\title{
Shape and deposition angle control of silver film-over-nanosphere SERS substrates
}

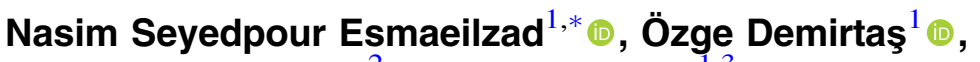 \\ Ahmet Kemal Demir ${ }^{2}$ (1) and Alpan Bek ${ }^{1,3}$ \\ ${ }^{1}$ Micro and Nanotechnology Program, Middle East Technical University, 06800 Ankara, Turkey \\ ${ }^{2}$ Department of Physics, Bilkent University, 06800 Ankara, Turkey \\ ${ }^{3}$ Department of Physics, Middle East Technical University, 06800 Ankara, Turkey \\ E-mail: e189101@metu.edu.tr
}

Received 8 July 2021, revised 3 September 2021

Accepted for publication 16 September 2021

Published 6 October 2021

\begin{abstract}
Thin metallic films on dielectric nanospheres are demonstrated to have a high potential for the fabrication of cost-effective SERS substrates. In addition to the morphological advantages that nanospheres offer for attaining a high density of hot spots, possessing shape adjustability by uncomplicated thermal treatment makes them an attractive platform for tuneable SERS substrates. Furthermore, when combined with the oblique angle metal deposition technique, adjustable gaps at a high density and adjustable shape of metal films, such as Ag films, can be achieved on nanospheres. Applying small changes in deposition angle can provide means for fine adjustment of the Raman enhancement factor $(\mathrm{EF})$, resulting in EF up to $10^{8}$ measured using crystal violet dye molecule as a Raman analyte. This practice paves the way for the fabrication of high EF SERS substrates at a reasonable cost using a monolayer of self-organized nanosphere patterns. An ultra-thin Ag film coated at $5^{\circ}$ tilt is shown to be an excellent substitute for a film deposited at $0^{\circ}$ with double the thickness. There is a strong agreement between the experimental results and finite-elements-method-based Maxwell simulations exhibiting expected field enhancements up to $10^{9}$ at a tilt angle of $5^{\circ}$.
\end{abstract}

Supplementary material for this article is available online

Keywords: nanospheres, enhanced Raman spectroscopy, oblique angle deposition, shape control

(Some figures may appear in colour only in the online journal)

\section{Introduction}

Raman spectroscopy is a highly sensitive optical characterization technique, which identifies the chemical components of a material employing inelastic scattering of incident electromagnetic radiation due to vibrations of chemical groups constituting the material. The Raman scattering, however, is a nonresonant low probability process as compared to resonant scattering processes such as elastic (Rayleigh) scattering or fluorescence. Inherently low signal/noise in Raman measurements leading to relatively high molecular detection limits have urged scientists to employ several signal amplifications schemes such as surface-enhanced Raman spectroscopy

* Author to whom any correspondence should be addressed.
(SERS) [1-4], resonant Raman scattering [5, 6], and coherent anti-Stokes Raman scattering (CARS) [7, 8]. Resonant Raman scattering and CARS techniques involve highly specific light sources such as tuneable lasers and a high level of experimental complexity; therefore, SERS stands out as the most widely utilized signal amplification technique in Raman spectroscopy. SERS has two main origins: chemical and physical enhancement [9-12]. Chemical processes such as charge transfer can lead to $10^{3}$ fold enhancement [13], where physical processes can lead to $10^{9}-10^{12}$ fold enhancement $[14,15]$ of the Raman signal. The physical enhancement in SERS is based on enhanced localized electromagnetic fields, which can be achieved by surface plasmon resonances (SPR) of metal nanostructures [1-4, 16]. Commonly, SERS enhancement factors (EFs) are found to be highest in nanostructured noble 


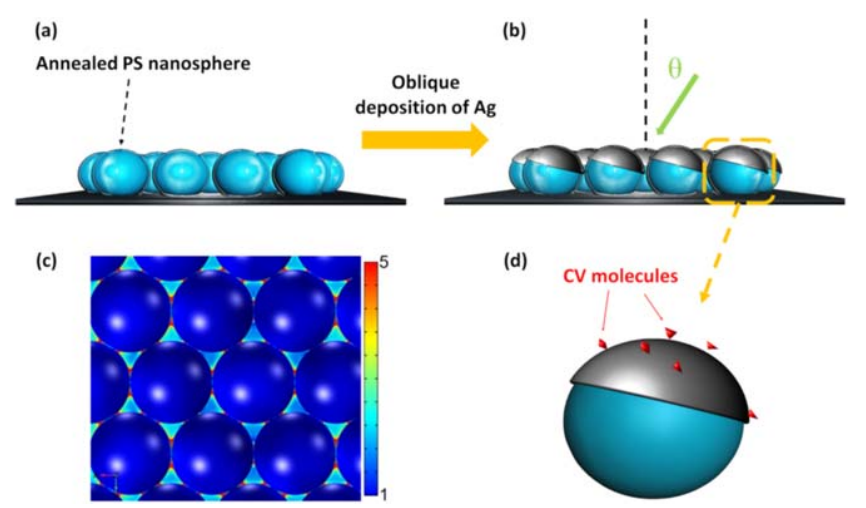

Figure 1. (a) Shape-modified hcp PS NSs on Si wafer, (b) Oblique angle deposition of an Ag film over PS NSs, (c) Top view of the Ag coated nanospheres depicting the field enhancement, (d) A simplified sketch of a nanosphere with the CV molecules (note: a continuous molecular coating is assumed in calculations).

metal film surfaces [17] or noble metal nano/microparticles that sustain strong field localization due to the presence of localized SPR (LSPR) and a high density of so-called hot spots $[18,19]$. Based on electromagnetic simulations such as Maxwell's equations solvers and experimental results, it can be concluded that EFs in SERS can be considerably improved if the LSPR band centre is close to the excitation wavelength [20-23]. Due to its straightforward integration into standard Raman spectroscopy systems, as simple as the replacement of standard substrates with SERS substrates, SERS has found its place in a wide variety of applications in chemical and biological sensing [24-27].

SERS substrates based on metallic structures can be fabricated using a wide variety of approaches, such as nanoparticle self-assembly [28], e-beam lithography [29], and nanosphere lithography (NSL) [30]. Among these methods, NSL has attracted considerable interest since it is a low-cost, robust, and reproducible method used for the fabrication of large area, ordered metal nanostructures. In NSL, hexagonally close-packed (hcp) polystyrene (PS) or silica nanosphere (NS) arrays are used as 2D masks to deposit metal nanostructures using thermal evaporation followed by removal of colloidal masks to form ordered triangular metallic nanostructures [31-34]. Another effective approach to achieve SERS substrates is keeping the colloidal NSL mask after metal evaporation. This is because the metal film on the nanospheres has a considerable roughness, which can improve the Raman signal substantially [35-41]. This method, also referred to as a metal film over nanospheres (FONs) or metal-capped nanospheres, can be employed for SERS detection of anthrax, glucose, biotin, and DNA [42-47].

The main privilege of the NSL method is that the tunability of the LSPR wavelength to the exciton wavelength is feasible as the plasmonic characteristics can be easily tuned by changing the size of nanospheres and thickness of the evaporated metal film [32]. There are several reports in literature targeting improvement or tunability of NSL based SERS substrate design. In one such work, it is targeted to increase the tunability of FON structures by deposition of $\mathrm{Ag}$ FONs on PDMS [48]. In another work, a proposed method for improvement is to coat the NSL mask with a bilayer of Ag and $\mathrm{Au}$ at the same thickness and determine optimized values for strong SERS signals [49]. In another study, the LSPR wavelength is tuned by using silica nanospheres with different diameters, but the thickness of $\mathrm{Ag}$ is constant [50]. Despite the practicality of such methods, it is beneficial to employ a technique that does not require different nanosphere diameters, and the LSPR wavelength shift can be achieved by Ag films over the NSL mask (AgFONs) with shape modification. In that respect, the oblique angle deposition method can pave the way to tune the LSPR wavelength by changing the vapor incident angle relative to the surface normal of the substrate. The Raman enhancement in such a structure is mainly due to the Ag film on the nanosphere rather than the underlying triangular islands [51].

Our study shows that a novel approach that combines two simple strategies can lead to attaining higher EF in SERS. Firstly, a single-size NSL mask is shape-modified using a simple and swift annealing-based technique to enhance electric field concentration, consequently leading to high SERS EFs [52]. Secondly, oblique deposition of $\mathrm{Ag}$ films on a shape-modified NSL mask, albeit at low tilt-angles, is employed for fine tuning the EFs. The idea of oblique angle deposition was previously used in [51] using larger $\theta$ resulting in not only a smaller Ag coating area but also in anisotropic thickness, which is not always desirable. Therefore, in our work, a custom-built thermal evaporation chamber is utilized to enable depositions at smaller angles, which enable fine adjustment of the LSPR wavelength.

Crystal violet $(\mathrm{CV})$, one of the most popular tracer dyes, was opted as the analyte to check the SERS response. Then, a relative EF is calculated by comparing the SERS spectra of $\mathrm{CV}$ acquired on $\mathrm{AgFON}$ substrates with $\mathrm{Ag}$ coated and bare Si wafers.

The SERS activity is measured using population statistics, which demonstrates that shape controlled AgFON monolayer substrates are very promising for a trace amount of molecule detection offering EFs up to $10^{8}$, which is in good agreement with the finite element method (FEM) based Maxwell solver simulations.

\section{Experimental methods}

\subsection{Preparation of AgFON arrays}

$2 \mathrm{~cm} \times 2 \mathrm{~cm}$ pieces of single-side polished silicon ( $\mathrm{Si}$ ) wafers are ultrasonically cleaned using acetone, isopropanol, and deionized water in sequence. A mild oxygen plasma treatment is carried out to make the surface of silicon samples hydrophilic. The solution of colloidal NSs, dispersed in water and functionalized with a hydroxyl group, was spin-coated on the Si samples. PS NSs self-organize in a hcp structure during the natural evaporation at room temperature, which is commonly used as the NSL mask in literature. Fabrication of NSL with this method yields about $5 \%$ of multilayers close to the sample edges and an overwhelming $95 \%$ of the central regions of the samples end in a monolayer structure. A low 
(a)

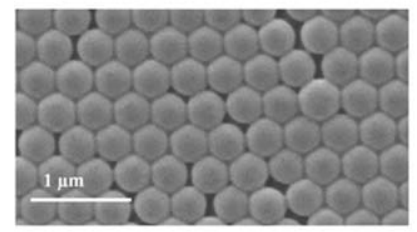

(b)

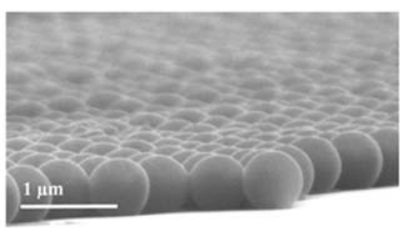

(c)

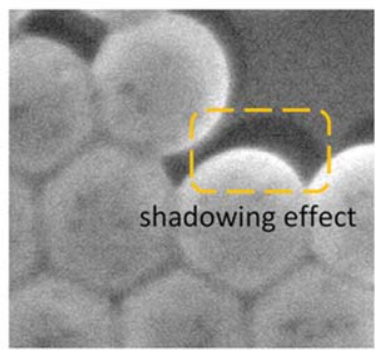

Figure 2. SEM images of (a) as-deposited hcp PS NSs with $350 \mathrm{~nm}$ size in top view, (b) $20 \mathrm{~min} 110{ }^{\circ} \mathrm{C}$ annealed PS NSs in side-view, (c) $5^{\circ}$ tilt $\mathrm{Ag}$ deposited annealed PS NSs showing the shadowing effect.

magnification SEM image is provided in the supplementary file (figure S1 available online at stacks.iop.org/NANO/32/505709/ mmedia) displaying the quality of large-area monolayers.

Subsequent thermal treatment of the NSL mask improves the adhesion of PS NSs to the Si surface. We have experienced that the NSs tend not to stay fixed on the surface of Si during coating of the dye molecules from aqueous solution unless this thermal treatment is employed, which is done at $110{ }^{\circ} \mathrm{C}$ for $20 \mathrm{~min}$ [52].

The diameters of PS NS employed for SERS substrates are $350 \mathrm{~nm}$. Ag films are deposited at two different thicknesses of $10 \mathrm{~nm}$ and $20 \mathrm{~nm}$ on a monolayer PS NS based NSL mask using a custom-built thermal evaporation deposition system at incidence angles of $\theta=0^{\circ}, 5^{\circ}, 10^{\circ}, 15^{\circ}$.

The fabrication steps are shown in figure 1. First, a PS NS-based NSL mask is fabricated and annealed at $110^{\circ} \mathrm{C}$ for 20 min on a hot plate (figure 1(a)). Next, thermal evaporationbased oblique angle Ag deposition is performed on the NSL mask, and fabrication of the AgFON array is finalized, as shown in figure 1(b). CV molecules are spin-coated on an AgFON array from an aqueous solution at $10^{-4} \mathrm{moll}^{-1}$ concentration. Figure 1(c) shows the field enhancement of nanospheres.

\subsection{Sample characterization}

The SEM images in figure 2 display the AgFON array structure viewed from different angles and at different magnifications. Figure 2(a) shows a top-view image of the AgFON array in which a uniform self-organized hep configuration of $350 \mathrm{~nm}$ size NSs is clearly visible. Figure 2(b) shows a side-view image of NSs after annealing for $20 \mathrm{~min}$ at a temperature of $110^{\circ} \mathrm{C}$. Figure 2(c) displays the shadowing effect due to tilted evaporation.

\subsection{SERS measurements}

A $10^{-4} \mathrm{M} \mathrm{CV}$ solution was used as an analyte for SERS measurements. AgFONs were spin-coated with $\mathrm{CV}$

molecules. The SERS signals were excited by using a linearly polarized continuous wave $(\mathrm{CW}) 532 \mathrm{~nm}$ wavelength laser source (Coherent Verdi) multimode (MM) fiber coupled to a modified upright microscope (Nikon Eclipse LV100) equipped with a $100 \mathrm{X} / 0.90 \mathrm{NA}$ objective. The excitation power is adjusted to $1.8 \mathrm{~mW}$ on the sample surface over a $17 \mu \mathrm{m}$ diameter round illumination spot. The incident polarization of the originally linearly polarized excitation laser is scrambled due to MM fiber delivery. The Raman signal was collected in epi-configuration by the same objective lens and coupled into another multimode fiber through a suitable dichroic mirror (Semrock) and a notch filter (Semrock). The Raman signals were analyzed by a f/9.8, $750 \mathrm{~mm}$ spectrometer (Andor Shamrock SR750) with $1501 \mathrm{~mm}^{-1}$ grating and an EMCCD camera (Andor Newton). The detailed explanation and setup sketch is provided in figure S2 in the supplementary file.

\subsection{Simulations}

COMSOL Multiphysics was employed to carry out FEMbased Maxwell simulations. A three-dimensional model of the AgFON was designed based on SEM images using COMSOL Multiphysics's CAD and Autodesk Meshmixer. The simulations were conducted to calculate the field enhancement of $\mathrm{AgFON}$ at different $\mathrm{Ag}$ deposition angle $(\theta)$ values from $0^{\circ}$ to $15^{\circ}$ (figure 4). The SEM images show that the Ag patch on each nanosphere is smaller than a half-circle. A linearly polarized CW plane wave at $532 \mathrm{~nm}$ wavelength is used in the simulations. The shape and coverage of the AgFON are specified considering the $\theta$ and the relative orientation of the NS monolayer domain with respect to polarization direction $(\varphi)$ of light. It is important to simulate different incidence polarization angles to ensure that the results are not specific to a single chosen configuration and the results adequately represent the experimental conditions. The dielectric function of $\mathrm{Ag}$ was taken from Johnson and Christy [53]. Further explanation regarding the simulation is provided in supplementary information along with figures S3 and S4. Figure 3 depicts the oblique deposition angles of $\theta$ of $0^{\circ}, 5^{\circ}, 10^{\circ}$ and $15^{\circ}$ with respect to the substrate as a sketch and simulation model (in grayscale).

The evaporation angle has a vital role to play as it can finely adjust the LSPR wavelength of AgFONs [38]. The optical response of AgFONs is dependent on $\mathrm{Ag}$ nanostructures and Ag film on NSs for small $\theta$. The larger the deposition angle becomes, the smaller the intersphere gaps become due to the shadowing effect. There is a critical $\theta_{c}$ with no nano triangles (around 55 ${ }^{\circ}$ ) [51]. As we target SERS due to $\mathrm{AgFONs}$ and not the triangular $\mathrm{Ag}$ nanostructures, we have carried out our experiments and simulations at smaller $\theta$ to study fine tuning. The simulation results of the maximum Raman EF as a function of polarization angle for deposition angle $\theta$ of $0^{\circ}, 5^{\circ}, 10^{\circ}$, and $15^{\circ}$ are shown in figure 4 . In the Raman EF calculations, we employ the well-accepted approximation of $\mathrm{EF}=E_{\mathrm{loc}}{ }^{4} / E_{0}{ }^{4}$ where $E_{\mathrm{loc}}$ and $E_{0}$ are the local and the incident field magnitudes, respectively. It is found that the Raman EF is maximized for $\theta=5^{\circ}$ for all incidence polarizations. 

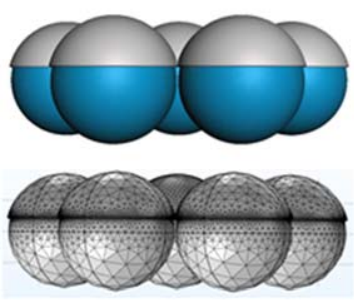

$\theta=0^{\circ}$

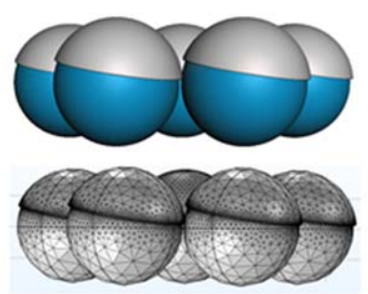

$\theta=10^{\circ}$
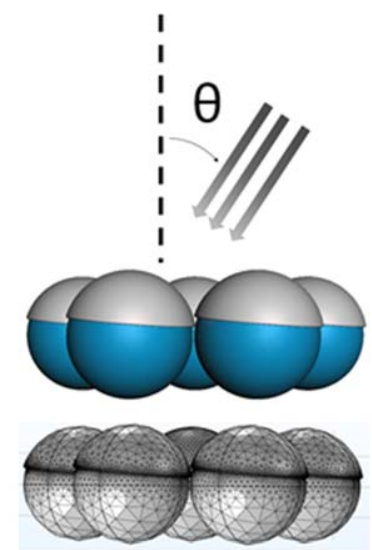

$\theta=5^{\circ}$

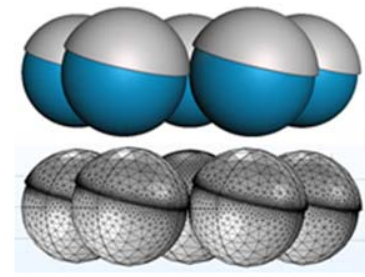

$\theta=15^{\circ}$

Figure 3. Sketch of the oblique deposition angle $\theta$ and the corresponding SERS structures.

When $\theta>5^{\circ}$, the EF has a considerable drop. The connection between EF and $\theta$ can be explained by the variation in the shape and coverage of Ag film, which takes place during oblique angle deposition. Dark field scattering spectroscopy measurements also display great agreement with FEM results, which can be seen in supplementary information figure S5.

\section{Results and discussion}

\subsection{EF calculation}

A unique definition of the $\mathrm{EF}$ is impossible due to experiment dependent situations such as single molecules, multiple molecules, experimental limitations, spatial distribution, orientations of the probe on the surface, etc. In fact, EF can strongly depend on the exact SERS conditions: substrate, analyte, excitation wavelength, etc [54]. Kamaliya et al predicted SERS EF from the hybrid gold-nanocone/graphene/ gold-nanohole tri-layers system via FDTD simulations. As nanocones are randomly spaced over the surface, three possible geometrical configurations are considered for the FDTD simulations: nanocone is- (i) far from the nanohole, (ii) at the center of the nanohole, and (iii) at the edge of the nanohole. For the configuration where the nanocone is at the edge of the nanohole, the maximum EF of $1.05 \times 10^{9}$ for $y z$-plane is achieved with $600 \mathrm{~nm}$ wavelength excitation [55]. Electromagnetic SERS EFs at hot spots are predicted to be on the

(a)

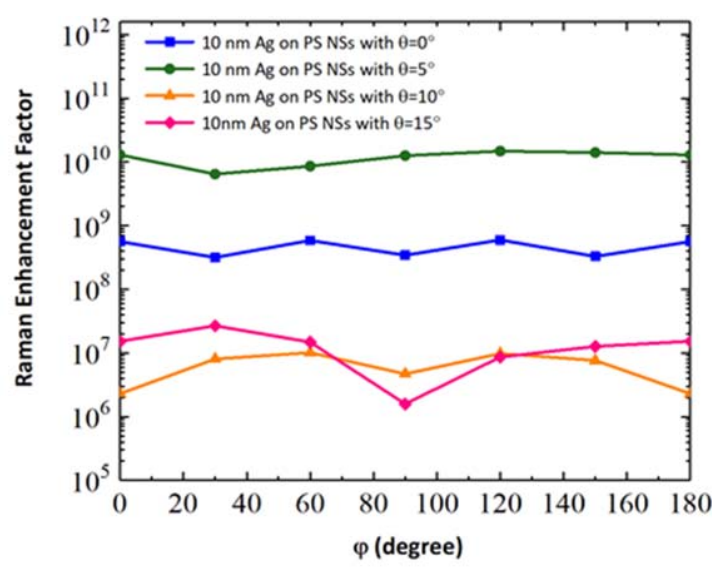

(b)

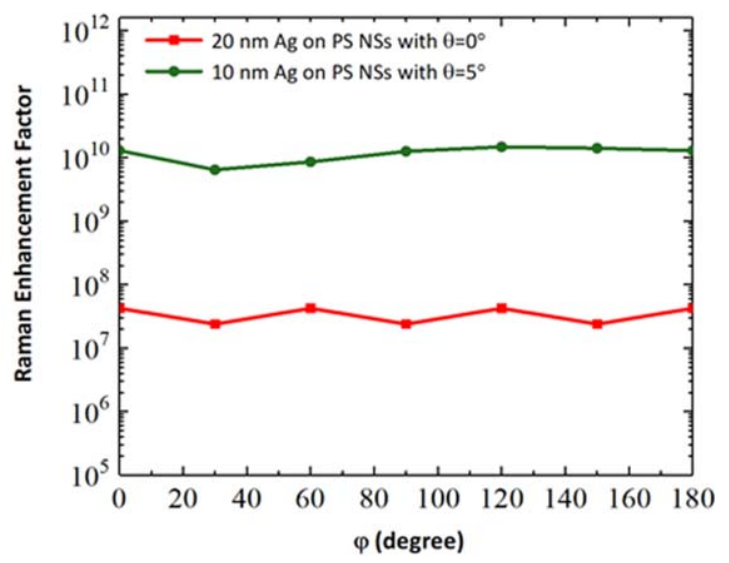

Figure 4. (a) Raman EF calculated using FEM simulations of AgFONs deposited at various $\theta$ as a function of incident polarization angle. (b) Raman EFs belonging to 20 and $10 \mathrm{~nm}$ Ag coatings at $0^{\circ}$ and $5^{\circ}$ of incidence.

order of $\sim 10^{10}-10^{11}$, for example, at a junction between metallic particles [54]. Because the single-molecule EF is the SERS enhancement on a given particular molecule at a specific point, it requires the exact definition of the SERS substrate geometry and the exact position and orientation of the probe on it [54]. Etchegoin et al performed single-molecule SERS detection of a nonresonant molecule, adenine, using an isotopically substituted adenine (N-adenine) as bianalyte SERS partners. They obtained maximum single-molecule EFs in the range of $7 \times 10^{10}-1 \times 10^{11}$ for adenine and $\mathrm{N}$-adenine, respectively [56]. However, the average SERS EF is calculated by averaging the signals expected for molecules randomly adsorbed over all possible positions on the metallic surface. Average SERS EFs are $\sim 10^{1}-10^{3}$ for non-optimized conditions, $\sim 10^{5}-10^{6}$ for 'standard' substrates, and $\sim 10^{7}-10^{8}$ for very good SERS substrates. Because the maximum singlemolecule EF only applies to one or a few localized regions of the surface, the average EFs are typically several orders of magnitude smaller. In addition, the average SERS EF is larger for more pointy structures, which increases with an increasing aspect ratio [57].

In normal Raman spectroscopy (NRS) inside liquid samples, the three-dimensional Raman probe volume can be determined by considering a prolate spheroid focal volume, which in our case have dimensions of $r_{x}=8.5 \mu \mathrm{m}, r_{y}=8.5 \mu \mathrm{m}$ and $r_{z}$ (depth 


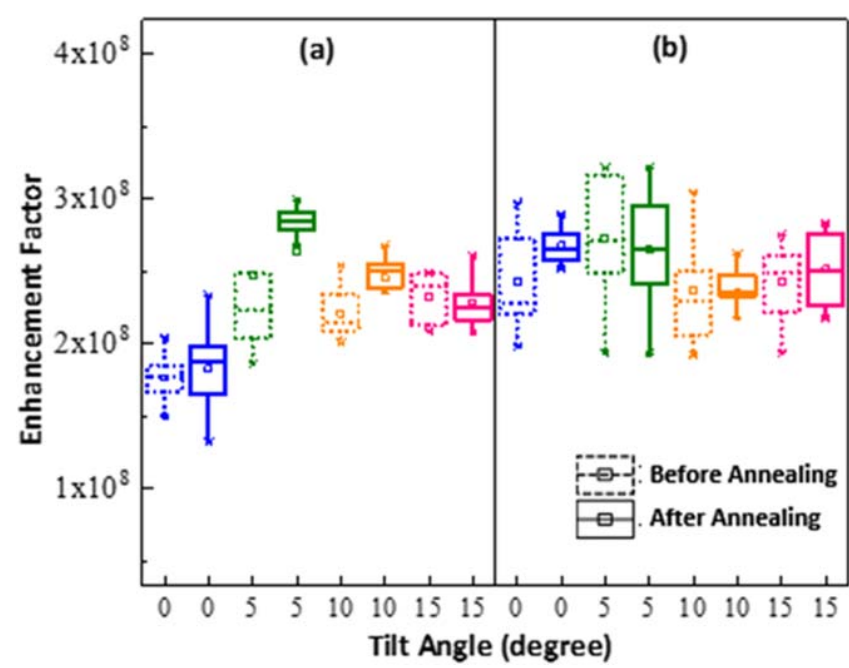

Figure 5. Box charts displaying the population statistics of SERS EFs of (a) $10 \mathrm{~nm}$ and (b) $20 \mathrm{~nm} \mathrm{Ag} \mathrm{coated} \mathrm{PS} \mathrm{NSs} \mathrm{before} \mathrm{(dotted}$ lines) and after (solid lines) annealing with $\theta$ values of $0^{\circ}, 5^{\circ}, 10^{\circ}$ and $15^{\circ}$.

of focus $)=17 \mu \mathrm{m}, \quad$ yielding $\quad V_{\text {probe }}=(4 \pi / 3) \times 8.5 \mu \mathrm{m}$ $\times 8.5 \mu \mathrm{m} \times 17 \mu \mathrm{m}=5144.88 \mu \mathrm{m}^{3}=5.14 \times 10^{-12} 1$ of probe volume. For our $10^{-4} \mathrm{M}$ solution, the total number of molecules $\left(N_{\mathrm{NRS}}=C_{\mathrm{NRS}} \quad X \quad V_{\text {probe }}\right) \quad$ in the measurement volume can be found as $N_{\mathrm{NRS}}=10^{-4} \mathrm{~mol} \mathrm{l}^{-1} \times 6.02 \times 10^{23}$ molecules $/ \mathrm{mol} \times 5.14 \times 10^{-12} \quad 1=3.09 \times 10^{8} \quad$ molecules. After baseline correction, $I_{\mathrm{NRS}}$ is calculated through the area under the peak $\left(1370 \mathrm{~cm}^{-1}\right)$ with a width between 1350 and $1386 \mathrm{~cm}^{-1}$ and is found as $322 \mathrm{cts} \mathrm{s}^{-1}$. Figure S6. in the supplementary file shows the SERS spectra together with the Raman spectrum of $\mathrm{CV}$ drop used for EF calculations. The Raman spectrum dominates the fluorescence of the CV molecule in the region of interest. In the estimation of the EFs, we considered a continuous molecular coating that extends to around $2 \mathrm{~nm}$ from the surface of the metal film while calculating the total number of $\mathrm{CV}$ molecules in the SERS measurements. In order not to overestimate the SERS EF, the number of molecules was multiplied further by a factor of 2 in order to ensure that the experimentally determined values approximate the minimum EFs exhibited by the substrates. Any discontinuous coating effects would mean higher actual EFs than the estimated ones.

The molecules in the near field extend to around $2 \mathrm{~nm}$ from the surface of the metal film, resulting in a probe volume of $V_{\text {probe }}=2 \mathrm{~nm} \times 227 \times 10^{6} \mathrm{~nm}^{2} \times 2=9.08 \times 10^{-16} 1$. The number of molecules in the near field was multiplied by 2 in order not to overestimate the EF. Therefore, the total number of $\mathrm{CV}$ molecules in the SERS measurement can be calculated as $N_{\text {SERS }}=10^{-4} \mathrm{moll}^{-1} \times 6.02 \times 10^{23}$ molecules $/ \mathrm{mol} \times 9.08 \times$ $10^{-16} 1=5.46 \times 10^{4}$ molecules. The EF for surface averaged structures are calculated with the measured INRS and ISERS values as described above. Figure 5 shows the experimentally determined EFs where statistics of SERS measurements cover spectra acquired from 10 different random spots on each substrate prepared at different $\theta$ and $\mathrm{Ag}$ coating thicknesses before and after annealing of PS NSs. Peripheral regions of the samples are avoided to ensure the uniform monolayer structure of the NSL.
Statistics show that maximum EF is obtained from $10 \mathrm{~nm}$ Ag coating with $\theta=5^{\circ}$ on annealed PS NSs, which is consistent with the simulation results. It is observed that the variation of EF versus tilting angle is expected from simulations to take place more pronounced when compared to experimental results. The difference can be attributed to the following reasons. Firstly, perfect spherical geometry is used in simulations. The sharpness of edges between gaps of spheres can cause very strong field intensities in simulations. However, in reality, the gaps exist between edges with edges of finite curvature, which have a limiting factor in achievable gap plasmon hotspot field intensity. A feature, which is most pronounced, especially in $\mathrm{Ag}$, is due to its tendency for dewetting the underlying surface. Furthermore, there are collective effects in this study, and it is impossible to consider all the factors in simulations. For instance, grain boundaries and dislocations between the arrays of nanospheres, which are inevitable, are not considered in simulation results. Dislocations partially originate from the finite size distribution of NS. Besides, evaporation begins in different locations and eventually unifies, which causes multi-domains. Despite our extensive optimizations, about $5 \%$ of the total area is coated as bilayers or multilayers. As described before, despite our efforts to avoid them, their presence cannot be completely excluded. Also, our focus here is shape and angle control, which is done using universal measurements. Since measurements are performed at large-area illumination spots from ten different locations, our results correspond to the average Raman EF rather than the maximum achievable values. The simulations, however, highlight the maximum achievable Raman EFs. In addition, experimental EF is influenced by many different factors, including film roughness, contributions of propagating SPPs, propagating and localized gap plasmons, singular hotspots, etc. Overall effects of these factors contribute to the experimental results. It is true that in some cases, surface roughness alone can create hotspots. Yet sometimes, true nanometer-scale roughness can act as a deterrent for strong propagating SPPs due to the considerable amount of scattering they cause. In the case when the surface roughness is at a tiny length scale to produce hotspots, it can still lead to SPP damping. No additional roughness than what the nanospheres produce is considered in our simulations. It is expected that the EF factors in simulations are higher than the experimental results. They should be used in order to expect a theoretical trend of the dependence of the EFs to the control variables of the experiment. It is evident that with increasing the deposition angle, the shadowing effect causes reduction of the Ag coating area and increase of the Ag film thickness variation.

Figure 5 shows that heat treatment-based shape control of PS NSs is a simple yet effective way to achieve homogeneous SERS substrates, as shown in the reduction of the population range in the box chart. Box charts show that not only does AgFON with $10 \mathrm{~nm}$ of thickness result in more homogenous SERS substrates but also enables significant material cost reduction. This is because by applying simple annealingbased shape control and oblique angle Ag deposition, we can achieve EFs even better than that of the AgFON with $20 \mathrm{~nm}$ 
thickness coated at a normal deposition angle. So, it can be said that at least half the amount of $\mathrm{Ag}$ material can be saved using this method. It is also evident from figure 5 that at a thickness of $20 \mathrm{~nm}$, the shape control, and tilt angle do not yield statistically significant changes in the EF. So, it can be said that these tuning parameters are most effective in achieving high EF SERS substrates with ultra-thin Ag layers. It should be noted that AgFON overlaps at borders of the adjacent nanospheres, i.e. the edges of AgFON touch rather than leaving small gaps between nanospheres. It is expected that as the angle of deposition inclines, it is more likely for nanogaps between the nanospheres to emerge (such as at $5^{\circ}$ of deposition angle). However, since the field strength decreases with increasing gap width, it is also expected for the field strength to decrease with a further inclination of the deposition angle (such as at 10o of deposition angle and above). In conclusion, we expect to observe an optimal angle of deposition which would generate maximum field enhancement. Assuming the gap effect to be the dominant mechanism for the $\mathrm{EF}$, the coating material can be minimized to a thickness that is sufficient to coat the nanosphere surface in a well-defined fashion to generate gaps in a controllable fashion. One can see that a similar gap effect also occurs for $20 \mathrm{~nm}$ thickness, yet the order of magnitude of EFs for $10 \mathrm{~nm}$ and $20 \mathrm{~nm}$ are the same for $5^{\circ}$ of deposition angle. So, one can save deposition material for the extra $10 \mathrm{~nm}$ of thickness.

For a clear understanding of the effect, the corresponding averaged SERS spectra are shown in figure 6(a). It is evident that at the same $\mathrm{Ag}$ coating thickness of $10 \mathrm{~nm}$, the Raman intensity can be enhanced around $10^{3}$ times on NSs with respect to flat $\mathrm{Ag}$ film on Si. Moreover, a shape adjustment of the NSs followed by an oblique angle $\mathrm{Ag}$ evaporation at $\theta=5^{\circ}$ can further improve the Raman signal substantially. Figure 6(b) clearly illustrates that with shape adjustment and oblique angle deposition, the necessary amount and hence the cost of $\mathrm{Ag}$ material can be cut by half. It should be noted that the consumed amount of evaporation material can easily be 3-4 orders of magnitude larger than the actual coated amount in thermal evaporators (depending on the source-sample distance).

\section{Conclusion}

In this study, annealing-based nanosphere shape adjustment and oblique angle deposition of Ag film have been utilized as a method to fabricate SERS substrates at a low-cost. The small variation in deposition angle enables fine tuning of the Raman enhancement factors of the AgFON structure. Raman enhancement factors as high as $10^{8}$ are achieved utilizing this technique from crystal violet dye molecule. This method enables cost-effective fabrication of SERS substrates with ultra-thin $\mathrm{Ag}$ films deposited at $5^{\circ}$ tilt can replace SERS substrates fabricated by deposition of Ag films with double thickness in normal deposition angle. FEM simulations show excellent agreement with the experimental results and demonstrate expected field enhancements up to $10^{9}$ at a tilt angle of $5^{\circ}$. This low-cost, high-performance SERS platform can be combined with further novel advances in the field [58]. (a)

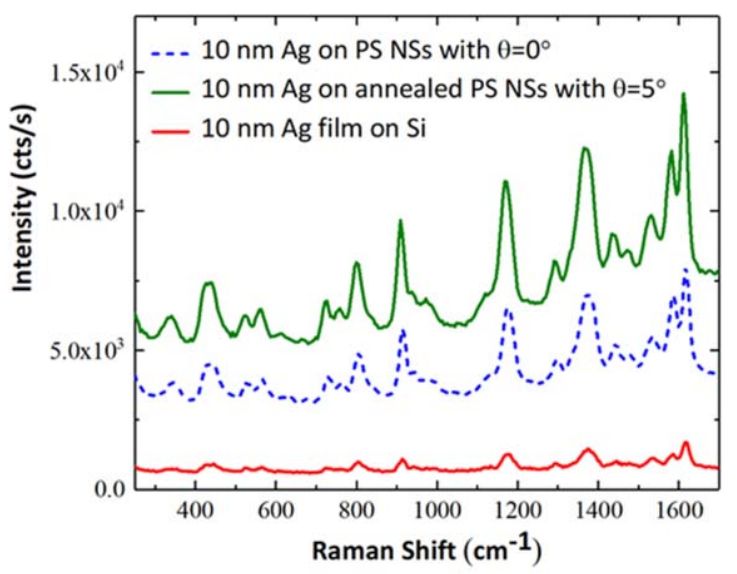

(b)

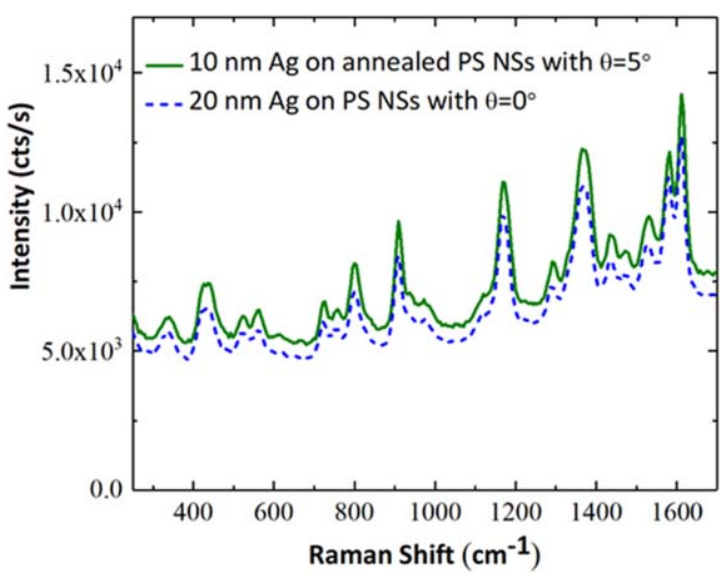

Figure 6. SERS spectra of $10^{-4} \mathrm{M} \mathrm{CV}$ spin-coated on (a) $10 \mathrm{~nm} \mathrm{Ag}$ on non-annealed PS NSs (dotted blue line), annealed PS NSs with $\theta=5^{\circ}$ (solid green line), and $10 \mathrm{~nm} \mathrm{Ag}$ coating on Si (solid red line), (b) $10 \mathrm{~nm} \mathrm{Ag}$ on annealed PS NSs with $\theta=5^{\circ}$ (solid green line) and $20 \mathrm{~nm} \mathrm{Ag}$ on non-annealed PS NSs without tilting (dotted blue line).

\section{Acknowledgments}

This work was supported by The Scientific and Technological Research Council of Turkey (TÜBİTAK) under grant nr 119F101. ÖD acknowledges The Scientific and Technological Research Council of Turkey (TÜBİTAK) 2211-C program and Turkish Council of Higher Education YÖK 100/2000 program for the scholarships. We thank Ahmet Oral for giving access to the Raman spectrometer and the microscope.

\section{Data availability statement}

All data that support the findings of this study are included within the article (and any supplementary files).

\section{ORCID iDs}

Nasim Seyedpour Esmaeilzad (ib https://orcid.org/00000001-6551-0296

Özge Demirtaş (D) https://orcid.org/0000-0002-1417-6491 
Ahmet Kemal Demir (10) https://orcid.org/0000-00023251-9793

Alpan Bek (10) https://orcid.org/0000-0002-0190-7945

\section{References}

[1] Dobson P J 2014 Introduction to metal-nanoparticle plasmonics, by Matthew Pelton and Garnett W. Bryant Contemp. Phys. 55 352-3

[2] Otto A 1983 Surface enhanced Raman scattering a otto Vaccum 33 797-802

[3] Lin W C, Huang S H, Chen C L, Chen C C., Tsai D P and Chiang H P 2010 Controlling SERS intensity by tuning the size and height of a silver nanoparticle array Appl. Phys. A 101 185-9

[4] Pérez-Mayen L, Oliva J, Torres-Castro A and De La Rosa E 2015 SERS substrates fabricated with star-like gold nanoparticles for zeptomole detection of analytes Nanoscale 7 10249-58

[5] Calleja J M and Cardona M 1977 Resonant Raman scattering in ZnO Phys. Rev. B 16 3753-61

[6] Renucci J B, Tyte R N and Cardona M 1975 Resonant Raman scattering in silicon Phys. Rev. B 11 3885-95

[7] Potma E O, Evans C L and Xie X S 2006 Heterodyne coherent anti-stokes Raman scattering (CARS) imaging Opt. Lett. 31 $241-3$

[8] Day J P R, Domke K F, Rago G, Kano H, Hamaguchi H O, Vartiainen E M and Bonn M 2011 Quantitative coherent anti-stokes raman scattering (CARS) microscopy J. Phys. Chem. B 115 7713-25

[9] Valley N, Greeneltch N, Van Duyne R P and Schatz G C 2013 A look at the origin and magnitude of the chemical contribution to the enhancement mechanism of surfaceenhanced Raman spectroscopy (SERS): theory and experiment J. Phys. Chem. Lett. 4 2599-604

[10] Xia L, Chen M, Zhao X, Zhang Z, Xia J, Xu H and Sun M 2014 Visualized method of chemical enhancement mechanism on SERS and TERS J. Raman Spectrosc. 45 533-40

[11] Yue W, Wang Z, Whittaker J, Lopez-Royo F, Yang Y and Zayats A V 2017 Amplification of surface-enhanced Raman scattering due to substrate-mediated localized surface plasmons in gold nanodimers J. Mater. Chem. C 5 4075-84

[12] Stoerzinger K A, Lin J Y and Odom T W 2011 Nanoparticle SERS substrates with 3D Raman-active volumes Chem. Sci. 2 1435-9

[13] Park W H and Kim Z H 2010 Charge transfer enhancement in the SERS of a single molecule Nano Lett. 10 4040-8

[14] Stiles P L, Dieringer J A, Shah N C and Van Duyne R P 2008 Surface-enhanced Raman spectroscopy Annu. Rev. Anal. Chem. 1 601-26

[15] Gwo S, Chen H Y, Lin M H, Sun L and Li X 2016 Nanomanipulation and controlled self-assembly of metal nanoparticles and nanocrystals for plasmonics Chem. Soc. Rev. 45 5672-716

[16] Dhawan A, Du Y, Yan F, Gerhold M D, Misra V and Vo-Dinh T 2010 Methodologies for developing surfaceenhanced raman scattering (SERS) substrates for detection of chemical and biological molecules IEEE Sens. J. 10 608-16

[17] Tian Z-Q, Ren B and Wu D-Y 2002 Surface-enhanced raman scattering: from noble to transition metals and from rough surfaces to ordered nanostructures Econ. Bot. $1069463-82$

[18] Ahmed W, Demirtaş Ö, Öztürk İ M and Bek A 2020 Monolayer assembly of multispiked gold nanoparticles for surface-enhanced raman spectroscopy-based trace detection of dyes and explosives ACS Appl. Nano Mater. 3 6766-73

[19] Staleva H et al 2009 Nanophotonics: plasmonics and metal nanoparticles Phys. Chem. Chem. Phys. 115866

[20] Kahraman M, Daggumati P, Kurtulus O, Seker E and Wachsmann-Hogiu S 2013 Fabrication and characterization of flexible and tunable plasmonic nanostructures Sci. Rep. 3 1-9

[21] Schatz G C, Duyne R P V, Zhao J, Dieringer J A and Zhang X 2008 Wavelength-scanned surface-enhanced resonance raman excitation spectroscopy J. Phys. Chem. C 112 19302-10

[22] Schlücker S 2014 Surface-enhanced raman spectroscopy: concepts and chemical applications Angew. Chemie - Int. Ed. 53 4756-95

[23] Kumar G V P 2012 Plasmonic nano-architectures for surface enhanced Raman scattering: a review J. Nanophoton. 6064503

[24] Fleischmann M, Hendra P J and McQuillan A J 1974 Raman spectra of pyridine adsorbed at a silver electrode Chem. Phys. Lett. 26 163-6

[25] Albrecht M G and Creighton J A 1977 Anomalously intense raman spectra of pyridine at a silver electrode J. Am. Chem. Soc. 99 5215-7

[26] Jeanmaire D L and VAN D R P 1977 Surface Raman spectroelectrochemistry :I. Heterocyclic J. Electroanal. Chem. 84 1-20

[27] Moskovits M 1978 Surface roughness and the enhanced intensity of Raman scattering by molecules adsorbed on metals J. Chem. Phys. 69 4159-61

[28] Kim B, Tripp S L and Wei A 2001 Self-organization of large gold nanoparticle arrays J. Am. Chem. Soc. 123 7955-6

[29] Abu Hatab N A, Oran J M and Sepaniak M J 2008 Surfaceenhanced Raman spectroscopy substrates created via electron beam lithography and nanotransfer printing ACS Nano 2 377-85

[30] And C L H and Duyne R P V 2001 Nanosphere lithography: a versatile nanofabrication tool for studies of size-dependent nanoparticle optics J. Phys. Chem. B 105 5599-611

[31] Colson P, Henrist C and Cloots R 2013 Nanosphere lithography: a powerful method for the controlled manufacturing of nanomaterials J. Nanomater. 2013 1-19

[32] Zhang X and Van Duyne R P 2005 Optimized silver film over nanosphere surfaces for the biowarfare agent detection based on surface-enhanced Raman spectroscopy Mater. Res. Soc. Symp. Proc. 876 207-12

[33] Zhang X, Yonzon M A, Stuart D A and Van Duyne R P 2005 Surface-enhanced Raman spectroscopy biosensors: excitation spectroscopy for optimisation of substrates fabricated by nanosphere lithography IEE Proc. Nanobiotechnol. 152 195-206

[34] Abdelsalam M E, Mahajan S, Bartlett P N, Baumberg J J and Rusell A E 2007 SERS at structured palladium and platinum surfaces J. Am. Chem. Soc. 129 7399-406

[35] Stropp J, Trachta G, Brehm G and Schneider S 2003 A new version of AgFON substrates for high-throughput analytical SERS applications J. Raman Spectrosc. 34 26-32

[36] Lin W C, Liao L S, Chen Y H, Chang H C, Tsai D P and Chiang H P 2011 Size dependence of nanoparticle-SERS enhancement from silver film over nanosphere (AgFON) substrate Plasmonics 6 201-6

[37] Nardelli M B et al 2008 Corrected 19 december 2008; see last page Science 321 388-93

[38] Xiao G N, Huang W B and Li Z H 2017 Rapid and sensitive detection of malachite green and melamine with silver film over nanospheres by surface-enhanced Raman scattering Plasmonics 12 1169-75

[39] Lee J, Zhang Q, Park S, Choe A, Fan Z and Ko H 2016 Particle-Film plasmons on periodic silver film over nanosphere $(\mathrm{AgFON})$ : a hybrid plasmonic nanoarchitecture 
for surface-enhanced Raman spectroscopy ACS Appl. Mater. Interfaces 8 634-42

[40] Flores-Romero E, Rodríguez-Sevilla E and Cheang-Wong J C 2018 Silver films over silica microspheres (AgFOSM) as SERS substrates Photon. Nanostruct. - Fundam. Appl. 28 81-7

[41] Baia L, Baia M, Popp J and Astilean S 2006 Gold films deposited over regular arrays of polystyrene nanospheres as highly effective SERS substrates from visible to NIR J. Phys. Chem. B 110 23982-6

[42] Farcau C, Giloan M, Vinteler E and Astilean S 2012 Understanding plasmon resonances of metal-coated colloidal crystal monolayers Appl. Phys. B 106 849-56

[43] Zhang X, Young M A, Lyandres O and Van Duyne R P 2005 Rapid detection of an anthrax biomarker by surface-enhanced Raman spectroscopy J. Am. Chem. Soc. 127 4484-9

[44] Ma K, Yuen J and Shah N 2011 In vivo, transcutaneous glucose sensing using surface-enhanced spatially offset Raman spectroscopy: multiple rats, improved hypoglycemic accuracy, low incident power, and continuous monitoring for greater than 17 days Anal Chem. 83 9146-52

[45] Lyandres O, Shah N C, Yonzon C R, Walsh J T, Glucksberg M R and Van Duyne R P 2005 Real-time glucose sensing by surface-enhanced Raman spectroscopy in bovine plasma facilitated by a mixed decanethiol/ mercaptohexanol partition layer Anal. Chem. 77 6134-9

[46] Himmelhaus M and Takei H 2000 Cap-shaped gold nanoparticles for an optical biosensor Sensors Actuators B $6324-30$

[47] Ngo H T, Wang H N, Fales A M and Vo-Dinh T 2013 Labelfree DNA biosensor based on SERS molecular sentinel on nanowave chip Anal. Chem. 85 6378-83

[48] Zhu X, Shi L, Liu X, Zi J and Wang Z 2010 A mechanically tunable plasmonic structure composed of a monolayer array of metal-capped colloidal spheres on an elastomeric substrate Nano Res. 3 807-12
[49] Wang J, Zhou F, Duan G, Li Y, Liu G, Su F and Cai W 2014 A controlled $\mathrm{Ag}-\mathrm{Au}$ bimetallic nanoshelled microsphere array and its improved surface-enhanced Raman scattering effect RSC Adv. 4 8758-63

[50] Greeneltch N G, Blaber M G, Schatz G C and Van Duyne R P 2013 Plasmon-sampled surface-enhanced raman excitation spectroscopy on silver immobilized nanorod assemblies and optimization for near infrared $\left(\lambda_{\mathrm{ex}}=1064 \mathrm{~nm}\right)$ studies J. Phys. Chem. C 117 2554-8

[51] Ingram W M, Han C, Zhang Q and Zhao Y 2015 Optimization of Ag-Coated polystyrene nanosphere substrates for quantitative surface-enhanced raman spectroscopy analysis J. Phys. Chem. C 119 27639-48

[52] Seyedpour Esmaeilzad N, Demir A K, Hajivandi J, Ciftpinar H, Turan R, Kurt H and Bek A 2021 Nanosphere concentrated photovoltaics with shape control Adv. Opt. Mater. 9 1-11

[53] Johnson P B and Christy R W 1972 Optical constant of the noble metals Phys. Rev. B 6 4370-9

[54] Le R E C, Blackie E, Meyer M and Etchegoin P G 2007 Surface enhanced Raman scattering enhancement factors: a comprehensive study J. Phys. Chem. C 111 13794-803

[55] Kamaliya B, Mote R G, Aslam M and Fu J 2019 Improved enhancement factor for SERS using broad ion beam induced self-organized gold nanocones MRS Adv. 4 697-703

[56] Le Ru E C, Blackie E, Meyer M and Etchegoin P G 2009 Single-molecule surface-enhanced raman spectroscopy of nonresonant molecules J. Am. Chem. Soc. 131 14466-72

[57] Le Ru E C and Etchegoin P G 2009 A quick overview of surface-enhanced Raman spectroscopy Principles of surface-enhanced raman spectroscopy ( Amsterdam: Elsevier Science) 1-27

[58] Postaci S, Yildiz B C, Bek A and Tasgin M E 2018 Silent enhancement of SERS signal without increasing hot spot intensities Nanophotonics 7 1687-95 